\title{
Expert Health Diagnosis and Monitoring System Software for Common Medical Conditions
}

\author{
M.O. Yinyeh \\ University for Development Studies \\ Faculty of Mathematical Science Computer Science \\ Department, Ghana
}

\author{
S. Alhassan \\ University for Development Studies \\ Faculty of Mathematical Science Computer Science \\ Department, Ghana
}

\begin{abstract}
The inadequate number of health personnel or professionals in some parts of the world especially in developing countries exposes patients to all manner of poor health service delivery by unqualified health personnel. To mitigate this phenomenon and reduce drug abuse in some cases, an expert health diagnosis system becomes handy to assist these unfortunate patients.
\end{abstract}

This research presents the design of expert medical system software used to diagnose common ailments of patients and prescribes. The software would present reliable diagnostic to improve accuracy and quality of healthcare in places where access to quality health is inadequate, inaccessible or not affordable. This system would also assist health professionals by providing them with information on diseases that they would not have suspected.

The software was developed using Visual Basic 2010 at the frontend and Microsoft SQL Server 2008 at the backend. The software can be installed on personal computers by medical practitioners, medical students or individuals who want to use this software to assist in administering first aid. The software works offline (it is not web based). The software minimum requirements for installations are; memory (RAM) $512 \mathrm{MB}$ and Hard disk drive 1GB.

\section{General Terms}

Monitoring System, Health Diagnosis, Expert System, Software

\section{Keywords}

Expert System, Software, Medical Condition, Diagnosis, Health

\section{INTRODUCTION}

The health of an individual is proportional to his or her productivity and life span. Therefore, a healthy population leads to a more productive country with a higher life expectancy rate. Minority of the populace know the common drugs and dosages that are supposed to be taken when they are not well and cannot easily access any medical facility or a medical practitioner in their neighborhood were illiteracy and poverty is very high. In such conditions, people tend to make their own diagnosis and then administer medicines based on their own prescriptions ignoring the consequences that would come afterwards [9]. This phenomenon is commonly practiced in rural (village) areas where medical facilities, staff and equipment's are inadequate or absent.

The most commonly abused drugs include pain killers and antibiotics. Patients who do not what to abuse these drugs visit health facilities with basic ailments such as slight cuts, bruises, headaches and the like, causing congestion or increasing the length of the queues in the few health post that are available in their locality, making it difficult for others with much more severe sicknesses to see the doctor.

Most patients do not know their Body Mass Index (BMI) or Blood Pressure (BP). This is one of the most basic medical vital information for diagnosis and treatment.

Research has contributed enormously to solve the problems of drug abuse, monitoring a person's health conditions (blood pressure, body mass index) and helping cure people of common diseases in places where medical facilities are inadequate, is still a challenge especially in developing countries [12].

This software would assist health practitioners, advice patients or users based on their blood pressure, body mass index and also offer assistance in administering common drugs (diagnosis) to cure patients with common diseases or medical condition. It will also have a system in place to remind users of medical review with their doctors. Diagnosis needs the integration of different sources of data and the on-line or offline collaboration of different kinds of specialists would be crucial for any software [8]. Using software that stores knowledge from these experts in the medical field would speed up accurate diagnosis processes. Some of the benefits that would be derived from medical diagnosis software include;

Increased availability of supervised administration of healthcare in rural areas

Reduced cost to accessing healthcare

Reduced the danger and level of drug abuse

Increase productivity or performance

Providing expertise to nurses and pharmacist to proper diagnosis and treatment where medical doctors are not adequate or absent.

Increased reliability to treatments for patients

This research therefore seeks to develop a database that stores basic ailment, prescriptions, BMI, BP and give advice based on the data provided by each patient.

\section{EXPERT SYSTEMS}

An expert system is a computer based application that performs task that is usually performed by human beings. These computer applications can be employed in various disciplines.

Expert systems were introduced by researchers in the Stanford 
Heuristic Programming Project, including the "father of expert systems" with the Dendral and Mycin systems. Some of the researchers who contributed to knowledge in this field includes; Bruce Buchanan, Edward Shortliffe, Randall Davis, William VanMelle, Carli Scott and some other scholars at Stanford. Expert systems at the time were the first accurately effective forms of Artificial Intelligence (AI) software developed over the period [5].

Artificial intelligence (AI) is the study of developing computer aided programs which exhibit human-like intelligence. An expert system is a computer program designed to model the problem solving abilities of human beings [1].

\section{EXPERT SYSTEM STRUCTURE}

The structure of expert systems is modelled after the human expert. Human expert need to understand and be able to solve the problem at hand and then uses specific information about the current problem to arrive at a solution. For example, a medical doctor with knowledge about possible diseases and information about a patient with symptoms will be able to provide a diagnosis to the disease of the patient. Expert systems have three main components that are; the knowledge base, working memory and inference engine [3].

\section{KNOWLEDGE BASE}

Knowledge Base is the part of an expert system that contains the facts, step by step procedure and rules needed to solve a particular problem; a coalition of facts and rules for problem solving is also termed Knowledge Base, these steps and rules are organized, stored in a database, shared, searched, and used. The knowledge base is a declarative representation of expertise, often in conditional statements like the IF THEN rules. These rules are logically related with each rule firing according to the working memory or the knowledge acquired in the course of running the program (software) [6].

\section{WORKING STORAGE OR MEMORY}

The working storage or memory refers to the data which is specific to a problem being solved by the system. The section of computer storage reserved for data to be temporarily stored during the running of a program to perform a task.

This data is provided during the execution of the program (software). Working memory is not always permanent as such, it is sometimes referred to as short-term (temporary) memory: The data is cleared when the execution is complete or the program exits [10].

\section{INFERENCE ENGINE}

An inference engine is a computer software system that is designed to draw conclusions by analyzing problems in light of a working database of designed and populated by an expert knowledge it draws upon

The inference engine refers to the source codes of the software developed which derives recommendations from the knowledge base and problem specific data in working memory to solve the problem.

The engine has two ways to run: batch or conversational. In batch, the expert system is equipped with all the necessary data to process from the beginning to the end. For the user, the program works as a classical system: the user provides data and receives results immediately. Reasoning for this system is invisible to the user. The conversational method becomes necessary when the software developer knows he/she cannot ask the user for all the necessary responses at the beginning, the problem being too complex. The software would have to reason to provide solution to the problem, request for the missing data from the user, gradually approaching the goal as swiftly as possible. The result gives the assertion of a discussion led by an expert, in this case the software. To guide a discourse, the engine may have several levels of complexity: "forward chaining", "backward chaining" and "mixed chaining" [2].

\section{FORWARD CHAINING}

Forward chaining is an assertion of new knowledge; all relevant inductive and deductive rules are fired comprehensively, efficiently making all knowledge about the current state overt within the state. Forward chaining as the name implies could be a progress from a known state towards a goal.

It is the investigation of a task or a problem without having an idea the final result or outcome and progressively work to obtain it, this takes several steps or stages to arrive at the solution (e.g. fault diagnosis) [13]

\section{BACKWARD CHAINING}

It refers to breaking down the steps of a task and teaching in reverse order. Backward chaining does not involve teaching a chain of events in converse order, as it seems to suggest by its name. It however involves supporting links in the chain, beginning at the rear of the chain and working toward the foundation, the system has a vivid understanding of the problem at hand. It starts from the end it hopes of getting the solution as soon as possible in a systematic manner, this principle is very necessary in developing expert software that would be efficient and easily diagnose problems that it would be confronted with by the users [13].

\section{MIXED CHAINING}

In mixed chaining, the system has an idea of the end result but it is not sufficient: it infers from forward chaining using previous responses and all that is possible before going ahead to the next question. So quite often the engine deduces the answer to the next question before asking it.

The paramount importance of implementing the system with logic is that this type of software is able to inform the user exactly what it is doing without any ambiguity and what it has deduced. Logic enables this form of system design quite easier and seamlessly, the most sophisticated and full-bodied expert systems are able to detect contradictions in user information or in the knowledge and can explain them plainly and at the same time, the expert's knowledge and its way of thinking clearly [11].

\section{SOME EXPERT SYSTEMS}

MYCIN, one of the popular diagnosis systems was developed for diagnosing only blood diseases. The short fall of this kind of expert system is that it cannot diagnose a cross section of diseases apart from that related to blood. It is one of the extensively researched expert systems because of its success. MYCIN was one of the pioneer expert systems to have employed production rules and to use the backward-chaining inference method to their development [7].

Production rules are IF-THEN statements that express chunks of knowledge that are readily applied to problem solving algorithms. Backward-Chaining refers to the search method used by the computer to look through the production rules and find the appropriate solution that fits the current problem. 
Many developers seek to use its suitability in expert systems to solve a variety of tasks and problems.

Over the years, researchers have developed several methods to increase the accuracy of computer aided diagnosis especially in medicine. This includes the use of probability theory and many others. For example, the Bayesian formula is used in medical field to determine the most likely disease a patient may have or suffer.

\section{MEDICAL DIAGNOSIS}

Medical diagnosis is a phrase that identifies as nearly as possible the specific clinical problem that is causing illness. This involves both the process of attempting to determine or identify a possible disease or disorder affecting a patient. Diagnostic process is a complex process which usually begins with the patient's own illness history and graduates into a result that can be grouped from one point to another depending on the symptoms gathered. This particular step in medicine is very important and significant to the treatment of every patient; this is therefore taking very seriously in the development and implementation of every expert or intelligent system in the medical field. If this module in the software development process is faulty then the system would not be efficient.

\section{DIAGNOSTIC PROCEDURE}

Diagnostic procedures are very important tools that aid physicians confirm or rule out the presence of a disorder or other medical condition in a patient.

A diagnosis can be termed as an attempt at classifying a patient's condition into blocks of finely distinct categories that allow a medical expert make a medical decision about treatment and diagnosis to be made. Subsequently, a problemsolving opinion is often described in terms of a disease or other condition but in the case of a wrong diagnosis, the patient's actual disease or condition is not the same as the individual's diagnosis made [4].

\section{SYSTEM DESIGN AND FUNCTIONALITIES}

\subsection{Database design}

The following are the tables of the database.

\subsubsection{BMI table}

This keeps track of the Body Mass Index (BMI) of the patients.

\subsubsection{Disease table}

This table stores the various diseases that the system will be able to recognize in order for complete diagnosis and recommendations.

\subsubsection{Drugs table}

This stores the various drug names, quantity to take a day and the minimum price of the drug. This is also needed for the recommendation.

\subsubsection{Login table}

This takes users information necessary for login into the system.

\subsubsection{Symptoms table}

This contains the various symptoms of all the diseases in the database.

\subsubsection{Users table}

The information about the full name of the user and other personal information are stored in this table.

\subsubsection{Drug disease table}

This table establishes the link between a disease and a drug. This is done by using the disease ID in the disease table and the drug ID in the drug table as foreign keys.

\subsubsection{Disease symptoms}

Each disease has certain signs and symptoms that show that the disease is present in a patient's body. This table shows the relation between a disease and a symptom. One disease may have several symptoms and one symptom may be related to several diseases. It takes disease ID and symptom ID as foreign keys on the table.

\subsubsection{Symptom synonym table}

This table shows other names by which a specific symptom may be referred to. It takes symptom ID and synonym ID as foreign keys.

\subsubsection{Full Medical History table}

This table contains the user's diagnosis history after each diagnosis. Patient ID is the only foreign key in this table.

\subsubsection{Medical history}

This links all the symptoms, diseases and drugs, BMI ID's to the user ID, Patient ID and medical history ID. All of these are foreign keys.

\section{ENTITY RELATIONSHIP DIAGRAM}

The database is reduced to the third nominal level. The relationships in the database are mainly one-to-many, manyto-one and many-to-many. Fig 9: shows these relationships

\section{HOW THE SOFTWARE FUNCTIONS}

The user is presented with the login form from which he/she can gain access to the software. New users are registered before they can gain access to the software.

The next step is the diagnosis; click the start button and complete series of questions presented by the software. Diagnosis module then generates a diagnosis from the responses presented by the user. Recommendation is then presented to the user.

When a common disease is found, the system will alert the user and then prescribe the appropriate drugs for that disease.

The unique feature of this software is its ability to diagnosis multiple or several diseases across all parts of the human body depending on the experts information and the correct symptoms presented to it by the patient or the user. 


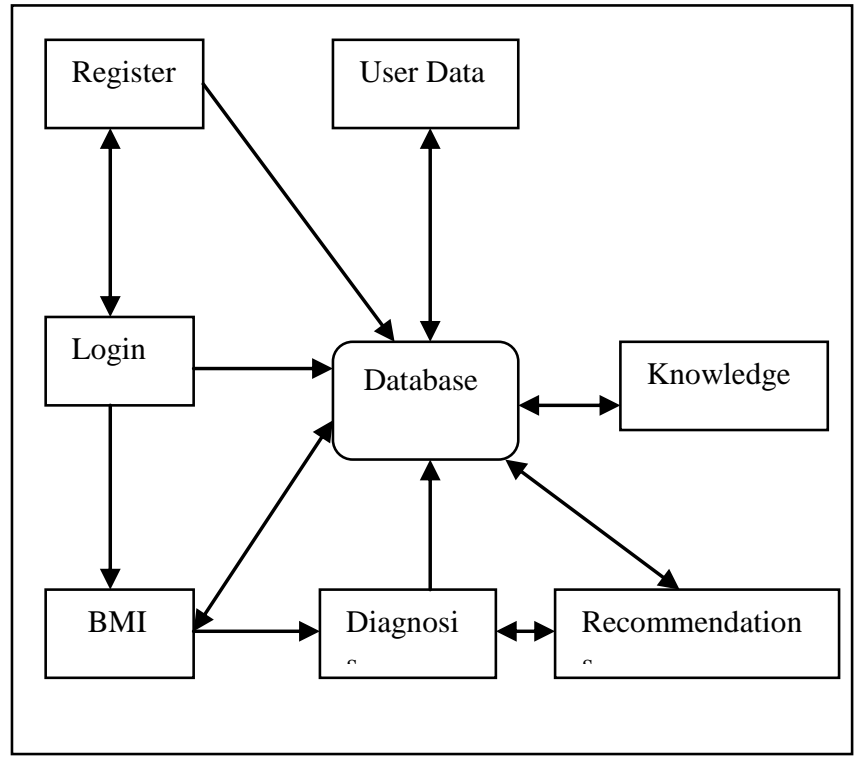

Fig 1: Functionality of the Software

\section{USER INTERFACE}

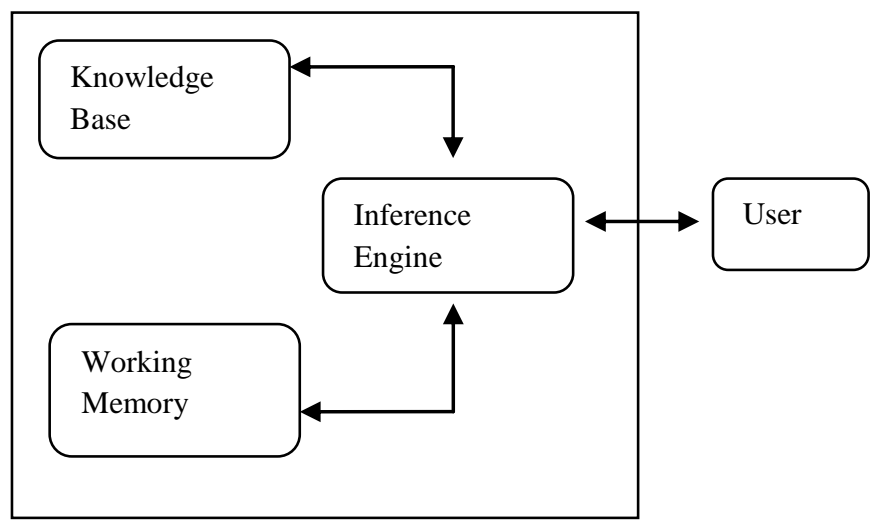

Fig 2: Expert system structure

Users are expected to login using their login details using fig 3 .

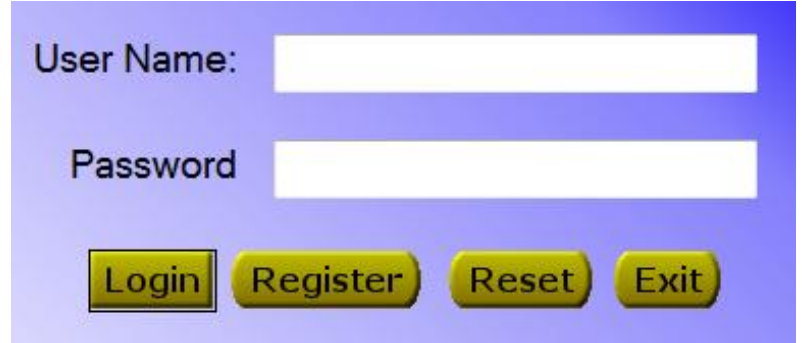

Fig 3: Login form for the software

When a user login is successful, he/she gains access to the administrative interface of the software as shown in fig 4 .

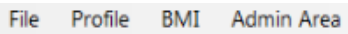

Please choose YES or NO to generate a diagnosis.

CLICK HERE TO START DIAGNOSIS PROCESS USING QUESTIONS

Please type your symptoms separated by comma

CLICK HERE TO ENTER YOUR SYMPTOMS

Fig 4: Administrative area of the software

After entering the symptoms of the patient, the user clicks on the diagnose button to generate the possible disease. This is illustrated in fig 5 .

Please enter your symptoms below separated by commas

PAINS. HEADACHE, FEVER

Diagnose Clear

Fig 5: Symptoms entry form

The software then matches the symptoms with various diseases symptoms and suggests the possible disease. This is shown in fig 6.

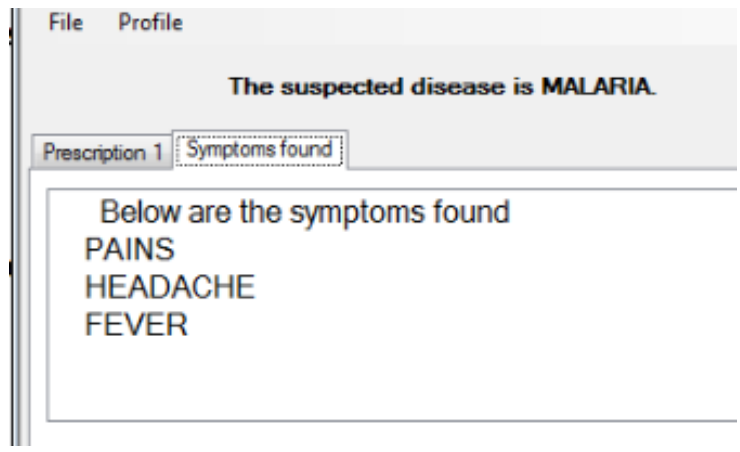

Fig 6: Diagnosis form

Fig 7, shows the drugs that would be given to the patient.

File Profile

The suspected disease is MALARIA.

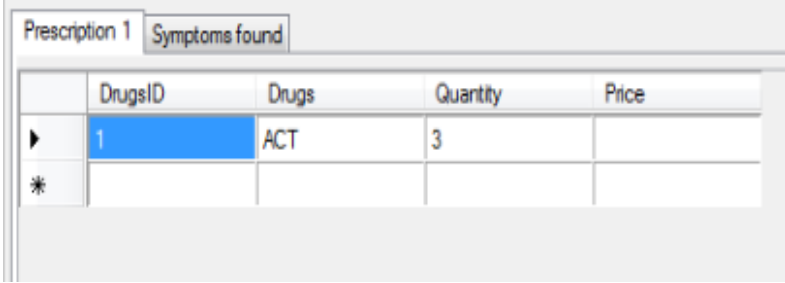

Fig 7: Prescription form 
Before the drugs are dispensed, the BMI of patients are considered. This shown in fig 8 .

$\begin{array}{ll}\text { BMI: } & 27.54821 \\ \text { Weight: } & 3 \\ \text { Height: } & 33 \\ \text { New Calculate Save Next }\end{array}$

Table 1, shows a section of results of patients BMI calculations made by the software.

Table 1: Results from the BMI Calculation

\begin{tabular}{|l|l|l|}
\hline BMI & Weight & Height \\
\hline 27.54821 & 3 & 33 \\
\hline 8.888889 & 5 & 75 \\
\hline 25.95156 & 3 & 34 \\
\hline 8.888889 & 5 & 75 \\
\hline 56.71077 & 3 & 23 \\
\hline 25.95156 & 3 & 34 \\
\hline 1.343725 & 2 & 122 \\
\hline
\end{tabular}

Fig 8: BMI Calculator form

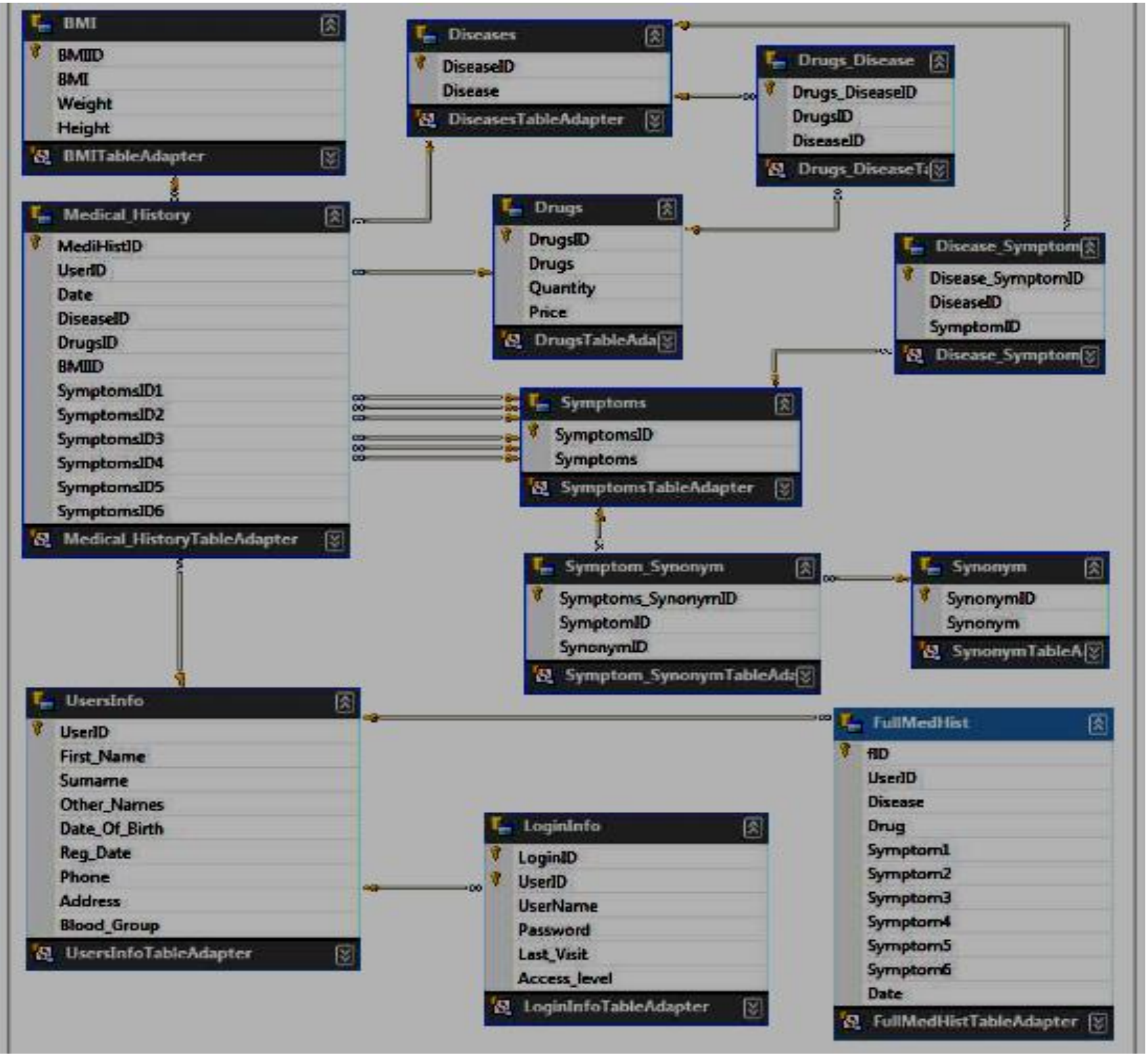

Fig 9: Entity Relationship diagram for the database 


\section{CONCLUSION}

A health diagnosis and monitoring software for common diseases across all parts of the human body has been developed.

The software would be a very good guide to individuals and health personnel in remote areas where medical personnel are inadequate.

It will also reduce the burden on hospitals, doctors and nurses while offering medical diagnosis at almost no cost to patients.

The software would further minimize drug abuse and improve quality of health delivery and reduce mortality in some situations.

Further research would be needed in the future to develop a web and mobile application version of this system.

\section{REFERENCES}

[1] A. F. Kana "Introduction to Artificial Intelligence Lecture Note" http://www.abu.edu.ng/maths/kana/cosc208.pdf

[2] Abhishek Paschisia, Akansha Awasthi, (2013) "Inference Engine" http://www.slideshare.net/AbhishekPachisia/inferenceengine

[3] Durkin, John, "Research Review: Application of Expert Systems in the Sciences" The Ohio Journal of Science. v90, n5 (December, 1990), 171-179 http://hdl.handle.net/1811/23417 https://kb.osu.edu/dspace/bitstream/handle/1811/23417/ V090N5_171.pdf

[4] E. Asif, (2013), "Exclusion of Diagnostic, Therapeutic and Surgical Methods from Patentability"
[5] G. Vidya, "Expert Systems and Decision Making" http://www.slideshare.net/akhilrocker143/572-11293384

[6] M Sasikumar, S Ramani, S Muthu Raman, KSR Anjaneyulu and R Chandrasekar, (2007) "A Practical Introduction to Rule Based Expert Systems" Narosa Publishers http://sigai.cdacmumbai.in/files/ESBook.pdf

[7] Nana Yaw Asabere, (2012) "mMES: A Mobile Medical Expert System for Health Institutions in Ghana" International Journal of Science and Technology, http://www.ejournalofsciences.org/

[8] Peggy Dettmer, Linda P. Thurston, Norma J. Dyck (2005) "Consultation, Collaboration, And Teamwork For Students With Special Needs, 5/E' http://www.ablongman.com/html/productinfo/dettmer5e/ 0205435238_ch2.pdf

[9] Productivity Commission 2005, Impacts of Advances in Medical Technology in Australia, Research Report, Melbourne

[10] RC Chakraborty, "Expert System" 2010, http://www.myreaders.info/07_Expert_Systems.pdf

[11] S.F. Nitodas , M.S. Ameen , L.M. Rubin, (2012) "Advantages of single and mixed species chaining for high productivity in high and mid-energy implantation" Ion Implantation Technology. 2002. Proceedings of the 14th International Conference

[12] Windber Medical center "2013 Community Health Needs Assessment" http://www.windbercare.org/chna2013.pdf

[13] Zahid Anwar, "Forward and Backward Chaining" 